\title{
Femtomolar Detection of Tau Proteins in Undiluted Plasma using Surface Plasmon Resonance
}

\author{
Suhee Kim $\dagger$, Alastair W. Wark $\ddagger$ and Hye Jin Lee* $\dagger$ \\ $\dagger$ Department of Chemistry and Green-Nano Materials Research Center, Kyungpook National \\ University, 80 Daehakro, Buk-gu, Daegu-city, 41566, Republic of Korea \\ \$ WestCHEM, Department of Pure and Applied Chemistry, Technology and Innovation Centre, \\ University of Strathclyde, 99 George Street, Glasgow, G1 1RD, UK \\ *Corresponding author: E-mail address: hyejinlee@knu.ac.kr; Tel. + 82053950 5336; Fax +82 \\ 053 950 6330; Postal address: Department of Chemistry and Green-Nano Materials Research Center, \\ Kyungpook National University, 80 Daehakro, Buk-gu, Daegu-city, 41566, Republic of Korea
}




\begin{abstract}
The ability to directly detect tau protein and other neurodegenerative biomarkers in human plasma at clinically relevant concentrations continues to be a significant hurdle for the establishment of diagnostic tests for Alzheimer's disease (AD). In this article, we introduce a new DNA aptamer/antibody sandwich assay pairing and apply it for the detection of human Tau 381 in undiluted plasma at concentrations as low as $10 \mathrm{fM}$. This was achieved on a multichannel surface plasmon resonance (SPR) platform with the challenge of working in plasma overcome through the development of a tailored mixed monolayer surface chemistry. In addition, a robust methodology was developed involving various same chip control measurements on reference channels to which the detection signal was normalized. Comparative measurements in plasma between SPR and enzyme-linked immunosorbent assay (ELISA) measurements were also performed to highlight both the 1000-fold performance enhancement of SPR and the ability to measure both spiked and native concentrations that are not achievable with ELISA.
\end{abstract}




\section{Introduction}

The establishment and sensitive detection of blood biomarkers that can be used as a reliable approach for the screening of neurodegenerative conditions such as Alzheimer's disease (AD) in a clinical setting continues to be a major challenge. ${ }^{1,2}$ Currently, the cost and time involved in diagnosing $\mathrm{AD}$ and other neurodegenerative conditions remains a major hurdle in tackling a condition which is rapidly increasing in priority for society. ${ }^{3}$ Efforts by researchers are hampered by a lack of accurate and sensitive analytical techniques capable of molecularspecific measurements in blood samples along with the growing need to compare the levels of different biomarker populations simultaneously. Amyloid- $\beta$ peptides and Tau proteins are consistently reported as promising serum biomarker candidates for AD patients along with a range of peripheral marker candidates connected to immunological mechanisms, inflammation and oxidative stress, and protein signaling pathways are also of interest. ${ }^{4-6}$ In the case of Tau protein, increased levels in AD patient plasma has been reported, ${ }^{7}$ however varying overlap in concentrations between different patient categories has prevented a plasma-based clinical screening tool being established.

Despite the limitations of the conventional enzyme-linked immunosorbent assay (ELISA) format, ${ }^{8,9}$ there have been very few alternative approaches reported capable of the rapid and direct detection of Tau in plasma. Recently, a single molecule ELISA digital array technology ${ }^{10}$ has been applied to the detection of femotmolar levels of Tau in blood. Surface plasmon resonance (SPR) has also been used to quantify the interaction between different Tau forms and antibodies ${ }^{11,12}$ as well as study DNA-Tau interactions, ${ }^{13}$ however no SPR measurements in plasma have been reported. Nanoparticles have been applied to create a localized SPR ${ }^{14}$ sensor as well as surface-enhanced Raman scattering ${ }^{15}$ and multi-photon scattering ${ }^{16}$ platforms but 
these studies were demonstrated in buffer only. Furthermore, all Tau diagnostic measurements reported to date have been limited to the use of either a single monoclonal antibody or an antibody pair in a sandwich assay format. Thus, the integration of nucleic acid aptamers ${ }^{17,18}$ as an alternative alongside robust platforms capable of multiplexed bioaffinity measurements would potentially have a major impact on AD research.

In this article, we report a new DNA aptamer/antibody pairing specific to Tau protein (Tau-381 isoform) for the first time and apply this assay on a multi-channel SPR platform for the direct detection of Tau in human plasma at femtomolar concentrations. Despite SPR now being a well-established technique for bioaffinity sensing, ${ }^{19-23}$ the quantitative detection of target proteins in plasma has proved challenging and among the recent examples published ${ }^{24,25}$ an even fewer number report analysis in plasma without significant dilution in buffer prior to measurement. ${ }^{26}$ Recently, we reported a new sandwich assay for another potential biomarker for $\mathrm{AD}, \alpha-1$ antitrypsin (AAT). ${ }^{27}$ As the native concentration of AAT is $\sim 40 \mu \mathrm{M}$, these measurements involved significant serum dilution in buffer prior to analysis. However, such an approach is not feasible for protein targets like Tau where concentrations in healthy patients can be in the femtomolar range. ${ }^{7}$ Thus, after establishing the DNA aptamer/Tau 381/antiTau sandwich assay performance in buffer, we also describe a robust methodology for the quantitative detection of Tau in plasma at clinically relevant concentrations. To achieve this, various mixed monolayer surface chemistries were compared in the optimization of the SPR chip as well as a series of non-specific controls simultaneously acquired on the same chips. Conventional ELISA measurements were also performed to support our results. 


\section{Experimental Section}

More extensive information on the materials used and the protocols related to SPR chip preparation, protein detection and also ELISA measurements are provided in the Supporting Information. In short, all in-situ SPR bioaffinity measurements were performed using a Biacore 3000. Recombinant Human Tau 381 full length protein (Tau) and tau antibody (antiTau) were acquired from Abcam. A 5'-amine modified DNA aptamer ${ }^{28}$ specific to Tau, whose sequence is 5'-H ${ }_{2} \mathrm{~N}-$ GCGGAGCGTGGCAGG-3' was purchased from IDT along with a control sequence, NC4, 5'-H2N-AGTACAAGAAGCTTACCAGCGAACTCAGTAGT CTGATATATATAACC$3^{\prime}$ which is actually specific to platelet factor $4 .{ }^{29}$ The proteins used for control measurements were recombinant human complement (CFH, R\&D Systems) and human complement factor $\mathrm{H}$ antibody (antiCFH, R\&D Systems). ELISA measurements were performed using a RayBio® human Tau ELISA kit (RayBiotech) featuring a 96 well microplate pretreated with antiTau in conjunction with a Sunrise-basic microplate absorbance reader (Tecan). For ELISA measurements, two different Tau proteins were used for the kit performance comparison; (i) Tau 381 human utilized in the SPR and (ii) Tau protein provided by the ELISA kit were used for ELISA measurements. All biomolecular interaction measurements were either performed in solution 1x PBS buffer pH 7.4 (Life Technologies) only, or directly in plasma from human (Sigma-Aldrich). When performing spiked measurements, different $\mu \mathrm{L}$ aliquots of the Tau stock concentration $(10 \mathrm{pM})$ of tau was directly added to plasma $(2-3 \mathrm{~mL})$ to prepare the desired spiked tau concentration.

\section{Results and Discussion}

SPR Detection of Tau Protein: It has been shown that Tau can bind to single-stranded DNA $(\mathrm{ssDNA})^{28}$ and double-stranded DNA (dsDNA). ${ }^{13}$ Indeed, it is understood that Tau protein 
plays a significant role in the protection of neuronal nucleic acids and that a combination of Tau deficiency and alterations (e.g. hyperphosphorylation) contributes to the DNA and RNA oxidation damage observed in Alzheimer's. ${ }^{30}$ However, while there have been several studies on Tau interactions with DNA sequences featuring several hundred bases, we found only one previous study demonstrating Tau affinity for a short DNA sequence, ${ }^{28}$ which, to our knowledge, has never been subsequently developed as an aptamer candidate for affinity-based sensing. Here, we demonstrate a previously unreported aptamer-antibody pairing for specific Tau detection.

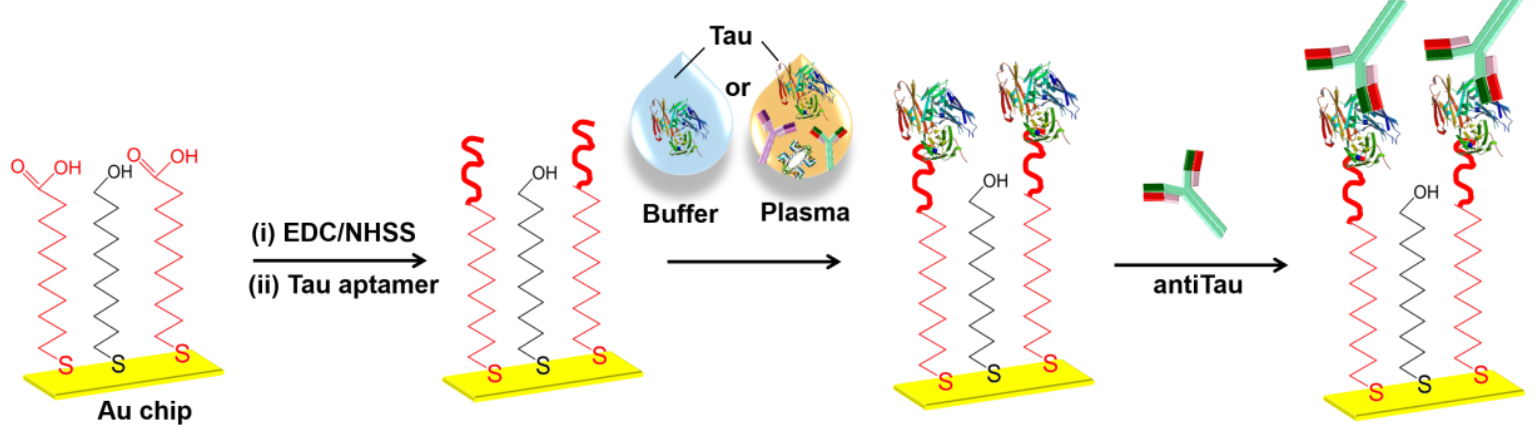

Figure 1. Schematic overview of strategy for Tau protein detection. Mixed monolayers of MUA and MUD were first immobilized on a SPR chip followed by the covalent attachment of DNA aptamer probes. Specific adsorption of Tau proteins was monitored in both buffer and plasma followed by the subsequent binding of antiTau. Images representative of Tau and CFH proteins are reproduced from references 31and 32, respectively.

An overview of the sandwich assay developed for plasma based detection is provided in Fig. 1. The SPR gold chip was first modified with a mixed monolayer of 11-mercaptoundecanoic acid (MUA) and 11-mercaptoundecanol (MUD). ${ }^{33}$ Both the Tau specific DNA aptamer and control sequences were covalently coupled to MUA via a $5^{\prime}$ end $\mathrm{NH}_{2}$ modification and EDC/NHSS linking chemistry. Tau specific adsorption measurements were first performed in buffer to establish the binding affinity of the DNA aptamer before progressing to measurements 
in plasma. Further enhancement of the SPR detection signal is then achieved by subsequently flowing antiTau (suspended in buffer) across the chip surface resulting in the formation of the surface sandwich complex. The covalent coupling of the DNA aptamer to the SPR chip surface is preferred to antibody coupling when there is a choice between both options. This is due to the better control over the probe molecule surface attachment, packing densities and nonspecific adsorption behavior that can be achieved using aptamer functionalization compared to antibody probes. ${ }^{27,34}$

Tau 381 is one of six known variants ranging from 352 to 441 amino acids in length. ${ }^{35}$ The relative abundance and molecular mechanism of each variant is still poorly understood and commercially available ELISA kits do not differentiate between variants, suggesting common epitope sites for antibody pairs across all species. For our investigation, we focused on tau 381 (Tau) based on the original study of Tau - ssDNA interactions by Krylova et al ${ }^{28}$ who also reported similar interaction behavior for the Tau 410 isoform. An initial assessment of the DNA aptamer - Tau interaction was first performed with the aptamer attached to a chip surface featuring only MUA rather than a mixed monolayer. A series of SPR measurements were performed monitoring Tau specific adsorption in buffer with the resulting change in reflectivity units ( $\Delta$ R.U.) obtained at different target concentrations plotted in Fig. 2a. This data was then used to create a plot of fractional surface coverage $(\theta)$ versus concentration, which was then fitted with a Langmuir adsorption isotherm model to obtain an adsorption coefficient ( $\left.K_{\text {ads }}\right)$ value of $6.3( \pm 0.1) \times 10^{8} \mathrm{M}^{-1}$. Comparative measurements were also performed using an antiTau modified SPR chip instead of the aptamer, which revealed a significantly lower $K_{\text {ads }}$ of $4.0( \pm 0.3) \times 10^{7} \mathrm{M}^{-1}$ (see Fig. S1 and Fig. S2 in the supporting information). This confirms the high affinity of the aptamer sequence for the Tau protein target and is at least comparable to 
other aptamer-protein interactions reported recently by us e.g. $K_{\text {ads }}=2.0( \pm 0.2) \times 10^{8} \mathrm{M}^{-1}$ for IgE protein-DNA aptamer ${ }^{36}$ and $K_{\text {ads }}=4.8( \pm 0.1) \times 10^{8} \mathrm{M}^{-1}$ for AAT protein-DNA aptamer affinity interactions. ${ }^{27}$

The utilization of mixed MUA:MUD monolayers was then explored. The motivation for this is based on recent reports that this approach promotes reduced non-specific adsorption of proteins,${ }^{37}$ plus DNA, peptide ${ }^{33}$ which is important for successful measurements in biofluids. A systematic study of the SPR sensing performance and also non-specific binding of bovine serum on mixed MUA:MUD layers has also been reported by Booksh et al, ${ }^{38}$ which highlighted the improvements that can be achieved using MUD. Shown in Fig. 2a are data series acquired for SPR chips prepared by initially incubating the bare gold surfaces with different molecular ratios of MUA and MUD prior to coupling the aptamer probe. As expected, smaller signals are observed when the surface density of the immobilized aptamer is lowered. For the 40\% MUD monolayer, the measured signal is only slightly lower than that obtained at $100 \%$ MUA coverage. A plot of fractional surface versus concentration is also shown in Fig. $2 \mathrm{~b}$ alongside the results from an experiment where 40\% MUD was instead replaced with 40\% PEG-SH. For comparative purposes, the largest signal change associated with the 100\% MUA measurements was utilized to calculate the fractional surface coverage of Tau at each monolayer composition. It can be clearly seen that a much lower signal is obtained for the $40 \%$ PEG monolayer than for $40 \%$ MUD, indicating that a better detection performance is obtained with the latter. The Langmuir isotherm fitting of the 100\% MUA chip is discussed above and the fitting of the $40 \%$ MUD, $60 \%$ MUD and 40\% PEG chips are shown in the supporting info, Fig. S2. A poorer fit quality was observed for the $40 \%$ PEG containing layer compared to all the MUA/MUD mixed layers tested as well as the $100 \%$ MUA layer. Representative SPR detection and non-specific 
control plots for both the MUD and PEG mixed monolayers are also shown in the supporting information where similar responses are observed. The poorer performance of the PEG layer observed throughout this work may thus be associated more with the organization of the mixed monolayer and the availability of the MUA end group for covalent coupling to the aptamer probe, with the PEG-SH being both longer and a different hydrophobicity than the MUD alkanethiol.
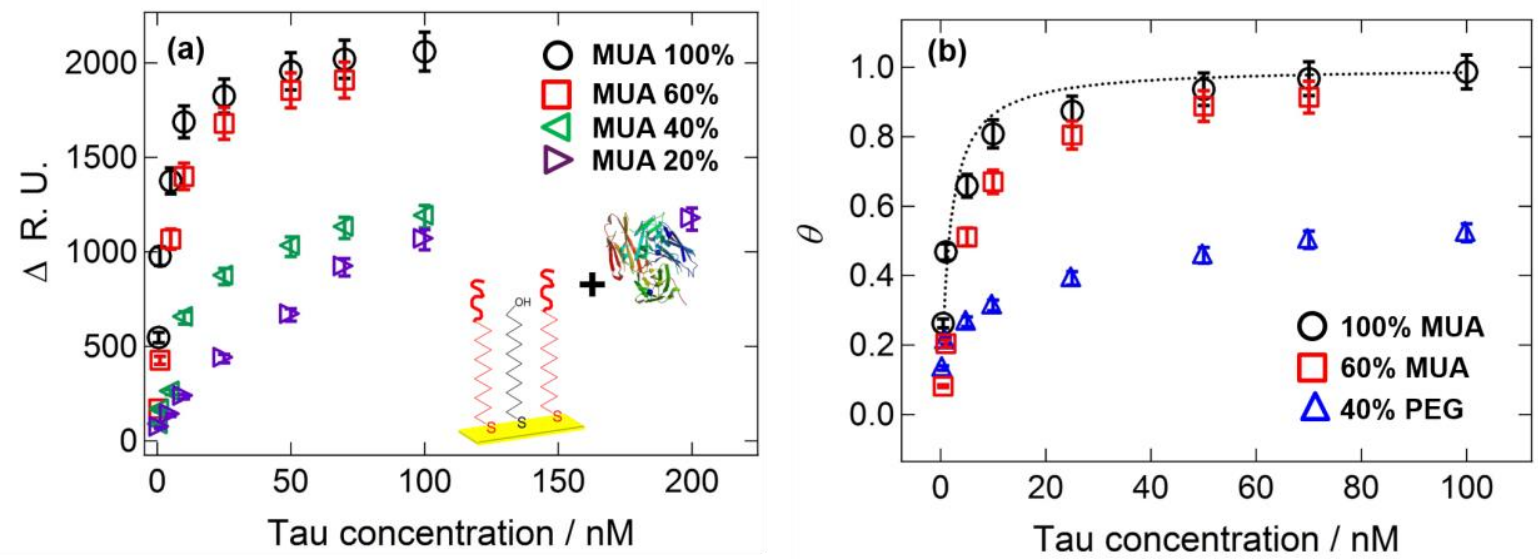

Figure 2. (a) Change in SPR signal obtained upon flowing a series of Tau concentrations in PBS buffer are compared for chip surfaces prepared with 100\% MUA and at MUA:MUD ratios of 60:40, 40:60 and 20:80. (b) Comparison of Langmuir adsorption isotherm plots showing fractional surface coverage ( $\theta$ ) versus Tau protein concentration for SPR chip surfaces with monolayers featuring $100 \%$ MUA, $60 \%$ MUA:40\% MUD and 60\% MUA:40\% PEG. In all three curves, the maximum signal change associated with the 100\% MUA surface is used for normalization.

Sandwich Assay: The formation of the surface sandwich assay was also first investigated in buffer solution using SPR chips prepared with different mixed monolayer compositions. Figure 3 compares a series of plots where antiTau is flowed across different SPR chips prepared with MUA:MUD ratios varying from 100:0 to 40:60 alongside a MUA:PEG ratio of 60:40. For each data point, the chip concerned was systematically exposed to a Tau concentration in the femtomolar range for at least one hour before injecting antiTau, which was kept at a fixed concentration of $100 \mathrm{nM}$. We have previously shown that the SPR response range and signal 
amplification also depends on the secondary probe concentration. ${ }^{27,39}$ The utilization of 100 $\mathrm{nM}$ antiTau in Fig. 3 is based on a series of measurements shown in Fig. S4 in the supporting information where different combinations of monolayer composition and antiTau concentrations are compared at a fixed Tau concentration. The results in both Figs. S3 and S4 indicate that the measurement dynamic range can be controlled via both the MUA:MUD ratio and the antiTau concentration with no significant improvement in sensing performance at antiTau concentrations above $100 \mathrm{nM}$. Importantly, these results also clearly confirm that the DNA aptamer and antiTau bind simultaneously to the Tau protein target via different epitope sites.

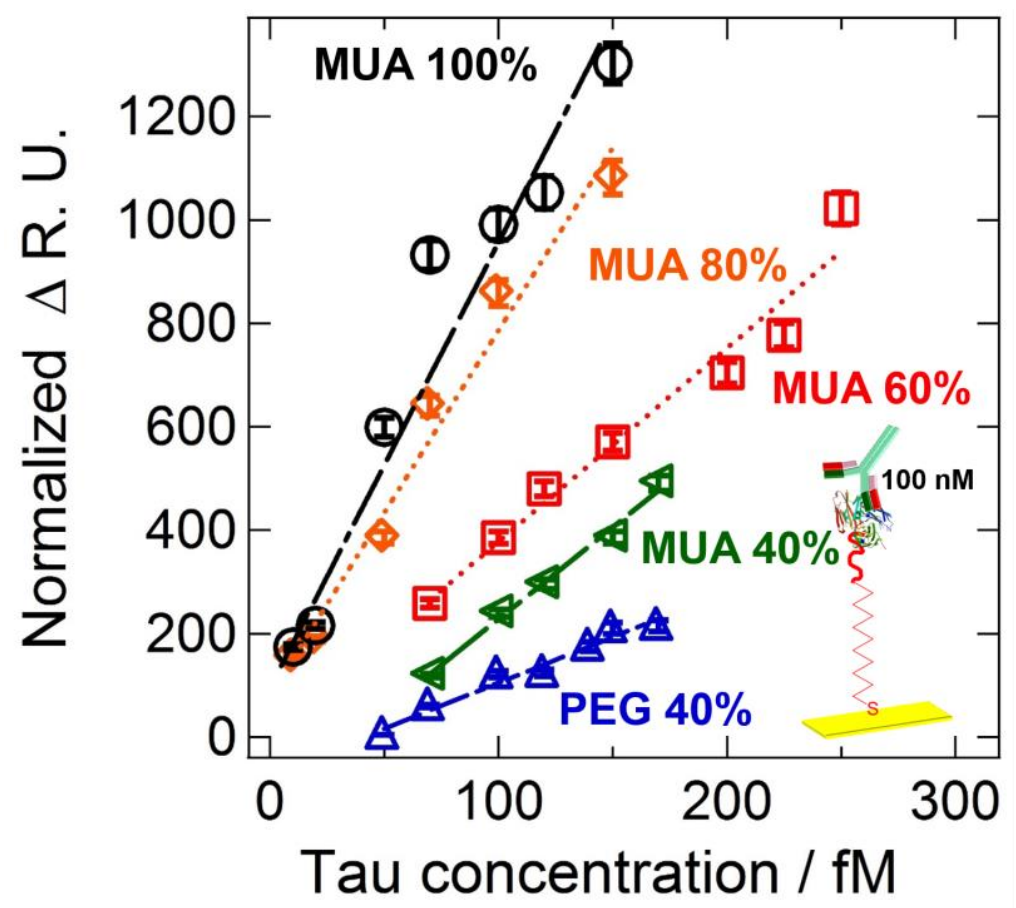

Figure 3. Normalized $\triangle R . U$. SPR responses monitoring the specific binding of antiTau onto chip surfaces that have been initially prepared with different MUA:MUD ratios (100:0 to 40:60) as well as MUA:PEG ratio of 40:60. Following attachment of the aptamer probe, each chip design was exposed to a different femtomolar concentration of Tau in PBS buffer, which was then followed by antiTau at a fixed concentration of $100 \mathrm{nM}$. The linear response range in each data series is highlighted by the dotted line. 
The normalized $\Delta$ R.U. values shown in Fig. 3 were obtained by acquiring the average $\Delta$ R.U. signals associated with specific target binding and subtracting this from an average of the nonspecific control signals, NC1 to NC4. These are summarized in Table 1, and involve repeat measurements in the absence of Tau (NC1), using a different target protein ( $\mathrm{CFH}, \mathrm{NC} 2)$, replacing antiTau with anti-CFH (NC3) or instead using a different aptamer sequence (NC4). For every data point presented in Fig. 3, control measurements were acquired utilizing two of the available 4 SPR chip channels with different controls used over the course of three repeat measurements to obtain an average signal. Representative NC measurements in buffer at different monolayer compositions are shown in Fig. S5 in the supporting information. In each case the NC SPR response is consistently much smaller than that of the measurements shown in Fig. S6. The fact that no consistent correlation in the relative intensities of control signals at different monolayer compositions was observed was another reason to use an average of the $\mathrm{NC}$ responses for normalization. We have found this methodology involving a series of simultaneous control measurements to be a robust approach to quantify the specific adsorption of the secondary antibody probe onto chip surfaces, especially where the target protein is present at low fractional surface coverages associated with femtomolar target concentrations. 


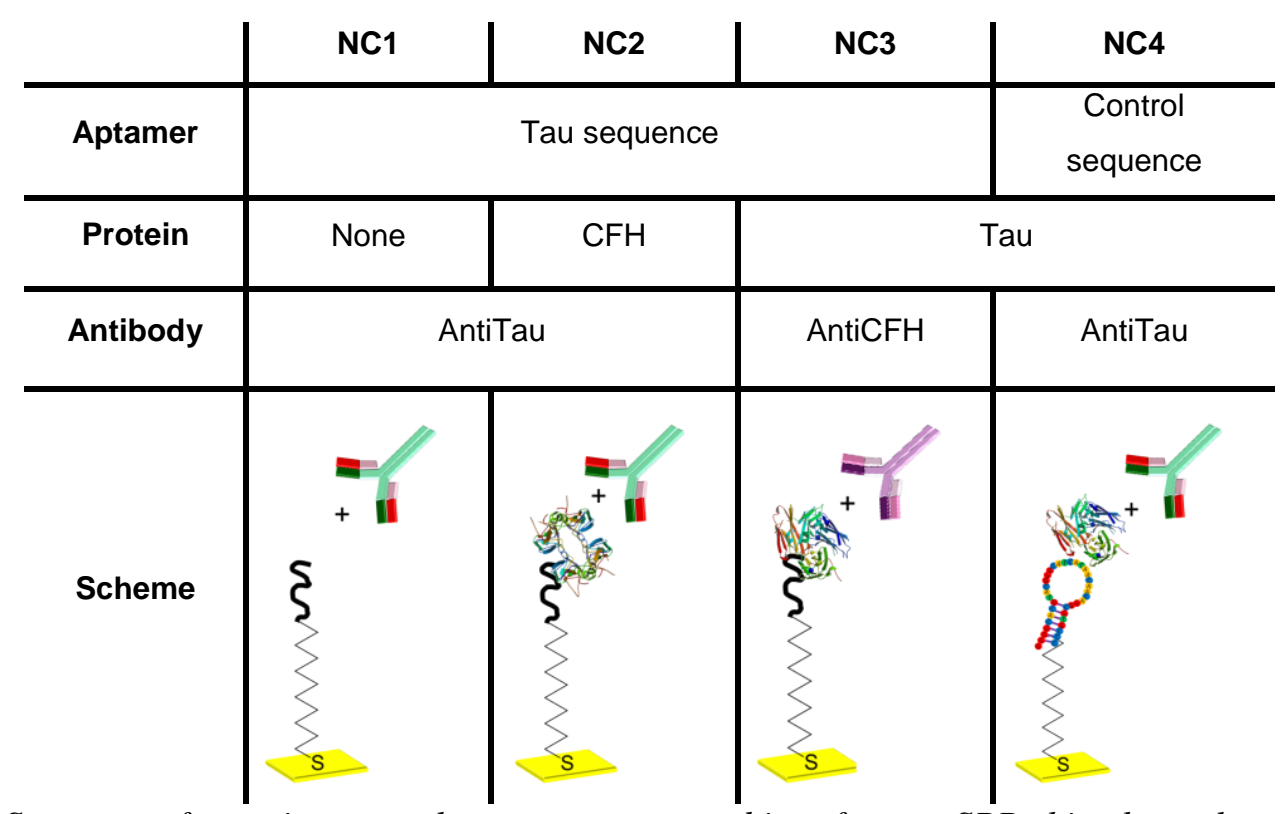

Table 1. Summary of negative control measurements used in reference SPR chip channels to which the Tau detection channel signals are normalized with respect to during the antiTau binding step performed in buffer.

Measurements in Plasma: Having established the Tau assay in buffer for different mixed monolayer compositions we then performed a series of SPR measurements directly in plasma alongside comparative measurements using a commercial ELISA sandwich assay kit and plate reader. Based on the optimization measurements described above, the antiTau concentration for the SPR sandwich assay was fixed at $100 \mathrm{nM}$ to enable femtomolar detection levels since the Tau concentration in normal patient plasma samples has been recently reported as low as 60-170 fM, respectively. ${ }^{7}$ The measurement dynamic range was further tuned by utilizing either 60\% MUA:40\% MUD or 40\% MUA:60\% MUD or 60\% MUA:40\% PEG monolayer surfaces.

Figure 4 shows a series of SPR results for different concentrations of Tau spiked into undiluted plasma for each of the three different monolayer compositions. Representative real-time RU plots are shown for spiked, non-spiked and negative control (NC) measurements along with the corresponding $\Delta$ R.U. and normalized $\Delta$ R.U. plots obtained for each data series. From the 
R.U. plots (Figs. 4a, 4d, 4g) it can be seen that both the spiked and non-spiked data curves are both consistently higher than the negative control data curves (see Fig. S7 for additional comparative plots). As expected, the R.U. signals for Tau-spiked plasma were consistently higher than the equivalent measurements performed in buffer. In order to clearly demonstrate the relative contributions of both non-specific adsorption of plasma proteins and also the specific adsorption of Tau to the larger signal, both $\Delta$ R.U. (Figs. 4b, 4e, 4h) and normalized $\Delta$ R.U. (Figs. 4c, 4f, 4i) plots were calculated. The results of linear fitting both are shown in Table S1 (supporting information) alongside the same analysis for the equivalent measurements performed in buffer earlier. The slope values for both the 60\% MUA:40\% MUD and 40\% MUA:60\% MUD spiking data sets show agreement between the plasma and buffer measurements. This indicates that the increase in the raw R.U. signal associated with nonspecific adsorption is generally uniform across all measurements and that the relative increases in signal between data points is due to a higher spiked Tau concentration. However, the $60 \%$ MUA:40\% PEG monolayer (Figs. 4g, h, i) exhibited a more limited concentration range where a linear response was observed. There was also a significant difference in the linear slope fits between the plasma and buffer data series for PEG (see Table S1, supporting information) as well as poorer SPR signal reproducibility, further highlighting the better performance of MUD compared to PEG. This is maybe due to the fact that the tightly and uniformly packed selfassembled monolayer of MUD mixed with MUA blocks nonspecific adsorption sites on the gold surface more effectively without impeding specific interactions. ${ }^{31,35}$

An estimation of the native Tau concentration initially present in the plasma was obtained by analyzing the $\Delta$ R.U. and normalized $\Delta$ R.U. plots. Considering the poor performance of the PEG containing layer, we focused on the MUA/MUD mixed monolayer chips here. For the 
$\Delta$ R.U. plots (Figs. $4 b$, e) the fixed y-offset value between the buffer and plasma series is due to a combination of both the presence of Tau alongside non-specific adsorption, while for the normalized R.U. plots (Figs. 4c, f) the offset between the two slopes in each case is primarily due to Tau specific adsorption. Also shown on all plots in Fig. 4 are measurements obtained for plasma only. These data points were compared with the analysis of the spiked Tau data series thus providing two approaches for evaluating the relative contribution of Tau specific adsorption to the overall SPR response signal. For the 60\% MUA chip, utilization of the linear slope fit for the normalized $\Delta$ R.U. series resulted in calculated native Tau concentrations of 50 $\mathrm{fM}$ (from spiked series analysis) and $35 \mathrm{fM}$ (from non-spiked plasma signal). Similarly for the $40 \%$ MUA chip, estimated values of $54 \mathrm{fM}$ and $52 \mathrm{fM}$ were obtained from the spiked and nonspiked data respectively (see Supporting info for more details). The good agreement between each of the data sets provides strong evidence of a native Tau concentration of $\sim 50 \mathrm{fM}$. This value is comparable with the only previous report that we are aware of that utilizes a sensitive analytical technique capable of demonstrating low femtomolar concentration levels of the target tau protein present in the plasma of healthy patients. ${ }^{7}$ In addition, it is interesting to note that for the $60 \%$ MUA chip, $\sim 45 \%$ of the SPR response was associated with Tau specific adsorption compared to $\sim 57 \%$ for the $40 \%$ MUA chip. Along with changes in surface probe density, this also contributes to the differences in the linear response range and SPR signal offsets observed between the two chips.

A final set of Tau detection measurements in both buffer and plasma were performed using a commercial ELISA kit and compared with the SPR surface sandwich assay. The ELISA results are shown in the supporting information (Fig. S8). A linear response detection range was obtained for spiked concentrations ranging from $2 \mathrm{pM}$ to $80 \mathrm{pM}$ in both buffer and plasma 
solutions with very close overlap in sensing performance in both environments. However, the ELISA performance was not sensitive enough to detect Tau present in non-spiked plasma. Further calibration assays were also performed comparing the use of the Tau provided by the ELISA kit manufacturer or instead using the same Tau 381 utilized in the SPR measurements. Interestingly, a steeper response slope was obtained for the Tau solution provided with the ELISA kit but no significant signal was detected below $2 \mathrm{pM}$ in either case. These measurements highlight the limited sensing performance of ELISA while SPR can be successfully applied to perform plasma diagnostics of native biomarker concentrations in the low femtomolar region. The improved performance of SPR compared to ELISA is associated with combination of several factors including better control over surface chemistry and nonspecific adsorption on the SPR chip and the use of continuous flow microfluidics beneficial at low target concentrations rather than static wells. A secondary binding step involving the formation of the sandwich assay is required to achieve SPR sensitivities in the fM range and the fact that this can be achieved directly for SPR while avoiding the larger errors associated with the enzymatic amplification reaction needed for ELISA signal transduction is also a significant advantage. 

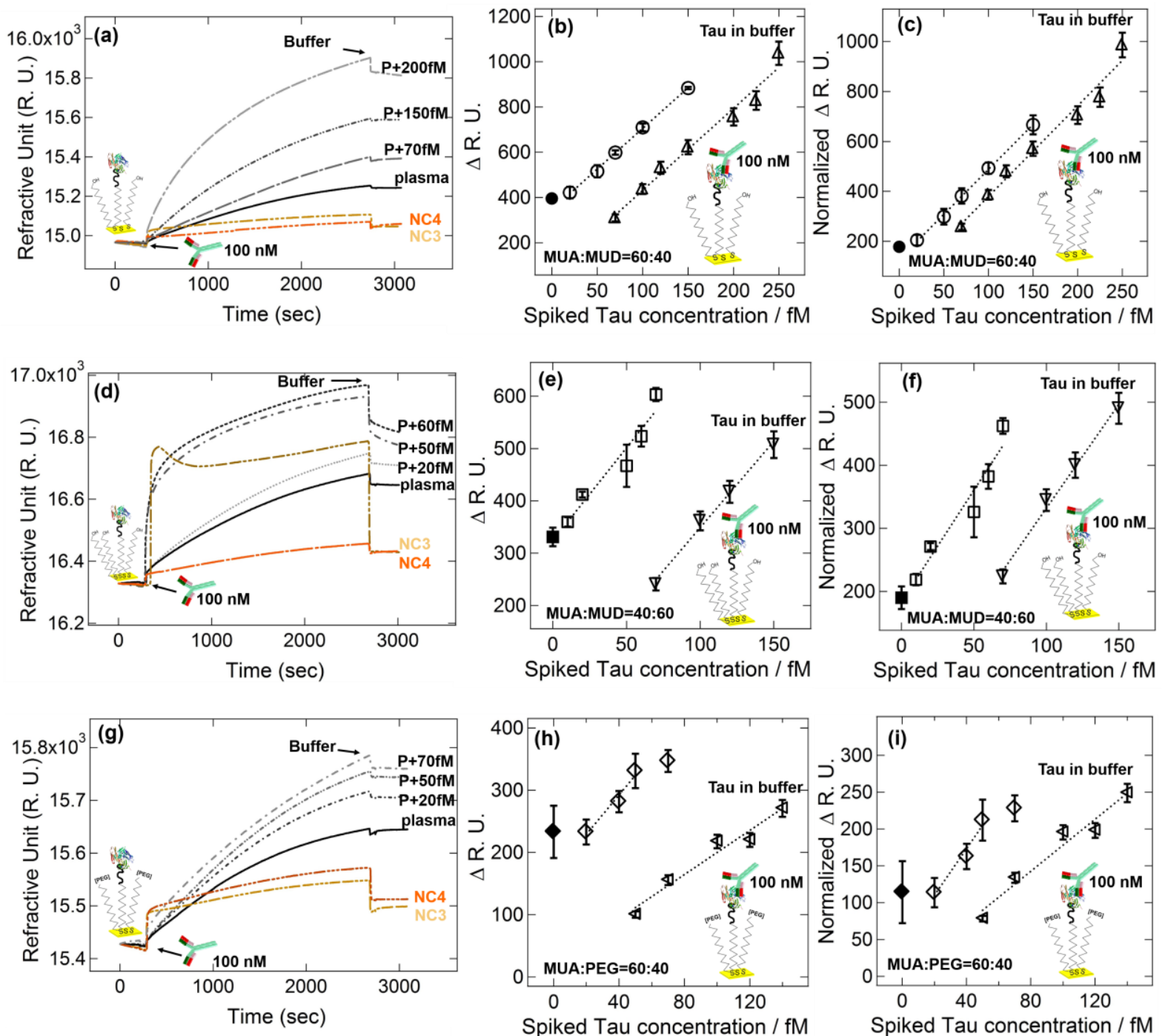

Figure 4. Analysis of SPR measurements in plasma for three different chip monolayer compositions: 60\% MUA:40\% MUD, 40\% MUA:60\% MUD, 60\% MUA:40\% PEG monolayer. (a), (d), (g) Real-time SPR measurements of antiTau adsorption following exposure to different Tau concentrations spiked directly into plasma, $P .(b),(e),(h) \Delta R . U$ plots obtained by calculating difference in R.U. signals in buffer before and after plasma exposure. $(c),(f),(i)$ is where the $\Delta R$.U signal has been normalized with respect to the average $\triangle R$.U. signal of all NC controls. The filled data point marker in the $\Delta R . U$ and normalized $\triangle R$.U. plots represents the plasma signal with no spiking. Representative NC3 and NC4 plots are also shown in $(a),(d)$ and $(g)$. 


\section{Conclusions}

In this article, a novel DNA aptamer-antibody sandwich assay specific to Tau protein biomarker has been developed and successfully applied for femtomolar concentration analysis in human plasma using surface plasmon resonance. The SPR chip surface chemistry was optimized via a series of experiments utilizing different ratios of a mixed monolayer of two differently terminated alkanethiols followed by the covalent coupling of the DNA aptamer in order to reduce the level of non-specific interactions while maintaining the analytical performance of the proposed surface sandwich assay. Robust control over the surface chemistry in combination with multi-channel measurement capability for simultaneous control measurements is essential for measurements in biological fluids such as plasma. Also, comparative measurements using ELISA for Tau analysis in plasma highlighted that the SPR based sandwich assay is a more sensitive option. As more important disease biomarkers are discovered and the corresponding nucleic acid aptamers selected, it is envisioned that the use of multiplexed SPR will continue to become more prevalent for medical diagnostics in biofluids.

\section{Acknowledgement}

This research was supported by the National Research Foundation (NRF) of Korea funded by the Ministry of Science, ICT, and Future Planning (grant number: NRF2013R1A2A2A03068833 and NRF-2015R1A2A1A15052198).

\section{Supporting Information}

Further experimental details include materials used, fabrication of aptamer and antibody biochips as well as SPR and ELISA measurements. Figures S1-8 are also included. This material is available free of charge via the Internet at http://pubs.acs.org. 


\section{References}

(1) Blennow, K.; Hampel, H.; Weiner, M.; Zetterberg, H. Nat. Rev. Nerol. 2010, 6, 131-144.

(2) Lista, S.; Faltraco, F.; Prvulvic, D.; Hampel, H. Prog. Neurobiol. 2013, 101-102, 1-17.

(3) Winblad, B.; Amouyel, P.; Andrieu, S.; Ballard, C.; Brayne, C.; Brodaty, H.; CedazoMinguez, A.; Bubois, B.; Edvardsson, D.; Feldmann, H.; Fratiglioni, L.; Frisoni, G. B.; Gauthier, S.; Georges, J.; Graff, C.; Iqbal, K.; Jessen, F.; Johansson, G.; Jonsson, L.; Kivipelto, M.; Knapp, M.; Mangialasche, F.; Melis, R.; Nordberg, A.; Rikkert, M. O.; Qiu, C.; Sakmar, T. P.; Scheltens, P.; Schneider, L. S.; Sperling, R. A.; Tjernberg, L. O.; Waldemar, G.; Wimo, A.; Zetterberg, H. Lancet Neurol. 2016, 15, 455-457.

(4) Schneider, P.; Hampel, H.; Buerger, K. CNS Neurosci. Ther. 2009, 15, 358-374.

(5) Sihlbom, C.; Davidsson, P.; Sjogren, M.; Wahlund, L.; Nilsson, C. L. Neurochem. Res. 2008, $33,1332-1340$.

(6) Song, F.; Poljak, A.; Smythe, G. A.; Sachdev, P. Brain Res. Rev. 2009, 61, 69-80.

(7) Zetterberg, H.; Wilson, D.; Andreasson, U.; Minthon, L.; Blennow, K.; Randall, J.; Hansson, O. Alzheimers Res. Ther. 2013, 5, 1-3.

(8) Lin, D.; Alborn, W. E.; Slebos, R. J. C.; Liebler, D. C. J. Proteome Res. 2013, 12, 59966003.

(9) Mayfield, S.; Lopata, A. L.; Branch, G. M. Mar. Biol. 2000, 137, 595-604.

(10) Rissin, D. M.; Kan, C. W.; Campbell, T. G.; Hows, S. C.; Fournier, D. R.; Song, L.; Piech, T.; Patel, P. P.; Chang, L.; Rivnak, A. J.; Ferrell, E. P.; Randall, J. D.; Provuncher, G. K.; Walt, D. R.; Duffy, D. C. Nat. Biotechnology 2010, 28, 595-600.

(11) Kontsekova, E.; Zilka, N.; Kovacech, B.; Skrabana, R.; Novak, M. Alzheimers Res. Ther. 2014, 6, 1-16.

(12) Yanamandra, K.; Kfoury, N.; Jiang, H.; Mahan, T. E.; Ma, S.; Maloney, S. E.; Wozniak, D. F.; Diamond, M. I.; Holtzman, D. M. Neuron 2013, 80, 402-414.

(13) Camero, S.; Benitez, M. J.; Cuadros, R.; Hernandez, F.; Avila, J.; Jimenez, J. S. PLOS ONE 2014, 9, 1-9.

(14) Vestergaard, M.; Kerman, K.; Kim, D.; Hip, H. M.; Tamiya, E. Talanta 2008, 74, 10381042.

(15) Zengin, A.; Tame, U.; Caykara, T. Biomarcromolecules 2013, 14, 3001-3009.

(16) Neely, A.; Perry, C.; Varisli, B.; Singh, A. K.; Arbneshi, T.; Senapati, D.; Kalluri, J. R.; Ray, P. C. ACS Nano 2009, 3, 2834-2840.

(17) Cho, E. J.; Lee, J.; Ellington, A. D. Annu. Rev. Anal. Chem. 2009, 2, 241-264.

(18) Zhou, W.; Huang, P. J.; Ding, J.; Liu, J. Analyst 2014, 139, 2627-2640. 
(19) Abbas, A.; Linman, M. J.; Cheng, Q. Anal. Chem. 2011, 83, 3147-3152.

(20) Ding, X.; Yan, Y.; Li, S.; Zhang, Y.; Cheng, W.; Cheng, Q.; Ding, S. Anal. Chim. Acta. 2015, $874,59-65$.

(21) Fasoli, J. B.; Corn, R. M. Langmuir 2015, 31, 9527-9536.

(22) Olaru, A.; Bala, C.; Jafferezic-renault, N.; Aboul-enein, H. Y. Crit. Rev. Anal. Chem. 2015, $45,97-105$.

(23) Sipova, H.; Homola, J. Anal. Chim. Acta. 2013, 773, 9-23.

(24) Piliarik, M.; Bockova, M.; Homola, J. Biosens. Bioelectron. 2010, 26, 1656-1661.

(25) Trapaidze, A.; Herault, J.; Herbert, J.; Bancaud, A.; Gue, A. Biosens. Bioelectron. 2016, $78,58-66$.

(26) Breault-Turcot, J.; Masson, J. Chem. Sci. 2015, 6, 4247-4254.

(27) Kim, S.; Lee, H. J. Anal. Chem. 2015, 87, 7235-7240.

(28) Krylova, S. M.; Musheev, M.; Nutiu, R.; Li, Y.; Lee, G.; Krylov, S. N. FEBS Lett. 2005, $579,1371-1375$.

(29) Jaax, M. E.; Krauel, K.; Marschall, T.; Brandt, S.; Gansler, J.; Furll, B.; Appel, B.; Fischer, S.; Block, S.; Helm, C. A.; Muller, S.; Preissner, K. T.; Greinacher, A. Blood 2013, 122, 272281.

(30) Violet, M.; Delattre, L.; Tardivel, M.; Sultan, A.; Chauderlier, A.; Caillierez, R.; Talahari, S.; Nesslany, F.; Lefebvre, B.; Bonnefoy, E.; Buee, L.; Galas, M. Front. Cell. Neurosci. 2014, $8,17-27$.

(31) Image from the RCSB PDB (www.rcsb.org): PDB ID 4TQE (Cehlar, O.; Skrabana, R.; Novak, M. 2015).

(32) Image from the RCSB PDB (www.rcsb.org): PDB ID 2G7I (Jaakola, V. P.; Jokiranta, T. S.; Goldman, A. Embo J. 2006, 25, 1784).

(33) Soler, M.; Estevez, M.; Moreno, M.; Cebolla, A.; Lechuga, L. M. Biosens. Bioelectron. 2016, 79, 158-164.

(34) Wu, B.; Jiang, R.; Wang, Q.; Huang, J.; Yang, X.; Wang, K.; Li, W.; Chen, N.; Li, Q. Chem. Commun. 2016, 52, 3568-3571.

(35) Goedert, M.; Jakes, R. EMBO J. 1990, 9, 4225-4230.

(36) Kim, S.; Lee, J.; Lee, S. J.; Lee, H. J. Talanta 2010, 81, 1755-1759.

(37) Briand, E.; Salmain, M.; Compere, C.; Pradier, C. Colloids Surf. B 2006, 53, 215-224.

(38) Masson, J.; Battaglia, T. M.; Cramer, J.; Beaudoin, S.; Sierks, M.; Booksh, K. S. Anal. Bioanal. Chem. 2006, 386, 1951-1959.

(39) Jang, H. R.; Wark, A. W.; Baek, S. H.; Chung, B. H.; Lee, H. J. Anal. Chem. 2014, 86, 814-819. 


\section{For TOC only}

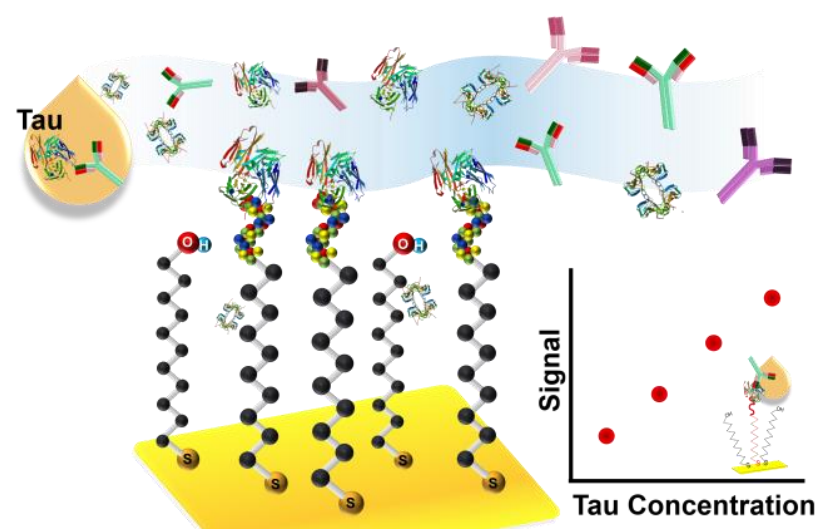

